\title{
Numerical and Experimental Investigation of Natural Convection in Open-Ended Channels with Application to Building Integrated Photovoltaic (BIPV) Systems
}

\author{
V. Timchenko ${ }^{1}$, O. A. Tkachenko ${ }^{1}$, S. Giroux-Julien ${ }^{2}$, C. Ménézo ${ }^{2}$ \\ ${ }^{I}$ The University of New South Wales Sydney NSW 2052, Australia \\ ${ }^{2}$ Centre d'Energétique et de Thermique de Lyon (CETHIL), CNRS-INSA-Univ. Lyon 1, Villeurbanne, France
}

\begin{abstract}
Numerical and experimental investigations of the flow and heat transfer in open-ended channel formed by the double skin façade have been undertaken in order to improve understanding of the phenomena and to apply it to passive cooling of building integrated photovoltaic systems. Both uniform heating and non-uniform heating configurations in which heat sources alternated with unheated zones on both skins were studied. Different periodic and asymmetric heating modes have been considered for the same aspect ratio $1 / 15$ of wall distance to wall height and for periodicity $1 / 15$ and $4 / 15$ of heated/unheated zones and heat input, $220 \mathrm{~W} / \mathrm{m}^{2}$. In computational study three dimensional transient LES simulation was carried out. It is shown that in comparison to uniformly heating configuration, non-uniformly heating configuration enhances both convective heat transfer and chimney effect.
\end{abstract}

\section{Introduction}

Building-integrated photovoltaic (BIPV) systems have been one of the fastest growing sectors of the renewable energy industry. In the context of green and energyefficient buildings, an attractive configuration is the double-skin system as illustrated in Figla. This arrangement consists of an air gap formed between the primary surface of a building and a secondary PV façade and can be designed vertically or tilted for rooftop applications. The double-skin BIPV system can not only supply electricity in-house to meet the demand of the building consumption, but also capture the heat from the incident solar radiation so that it can be used to reduce the heating load of the building during winter times. However, as pointed out by Bloem [1], when exposed to intense solar radiation, the temperature of the PV modules increases because not all of the incident radiation is converted into electrical energy. As a result, the efficiency decreases significantly

To overcome this problem the double-skin configuration can provide passive cooling of BIPV systems by means of natural convection of air which is entrained in the open-ended channel formed by a gap between the walls.

To investigate processes inside BIPV systems a considerable amount of research has been undertaken on uniformly-heated open channels [2-5]. The configuration with variable parameters, including the channel inclination angle, aspect ratio, and different values of the electric heat flux on boundaries, with two- or one-sided heating has been considered. At the same time, staggered configuration such as demonstrated in Figure $1 \mathrm{~b}$ has been shown by Fossa et al. [6] to achieve better cooling of the panels inside the channel. In such configuration, the outer skin is composed of alternating PV modules (localized heat sources) and glazed panes (unheated zones) over its entire height. Similarly, the inner skin has alternating windows (heat sources) and shaded wall (unheated zones).

These configurations may also be more practical for BIPV applications such as buildings with windows. In order to simulate actual situations, uniformly heated strips alternating with unheated strips on each side of the channel, as shown on figure $1 \mathrm{~b}$, were studied experimentally in CETHIL, Lyon and UNSW, Sydney [6-7]. It was shown that for a given channel heat flow and channel aspect ratio, staggered configurations promote better heat transfer, consequently reducing the maximum wall temperature compared to a uniformly heated wall [6$8]$.

In this paper both numerical and experimental investigations of the effects of the non-uniformity of the wall heat flux distribution on the natural convective flow and heat transfer within a vertical rectangular open ended channel are presented and compared with results obtained for the uniformly heated configurations.

\footnotetext{
${ }^{\text {a }}$ Corresponding author: v.timchenko@unsw.edu.au
} 


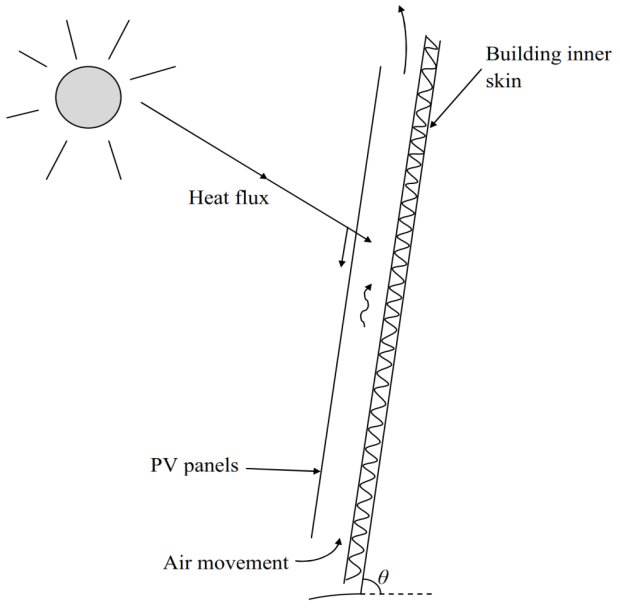

(a)

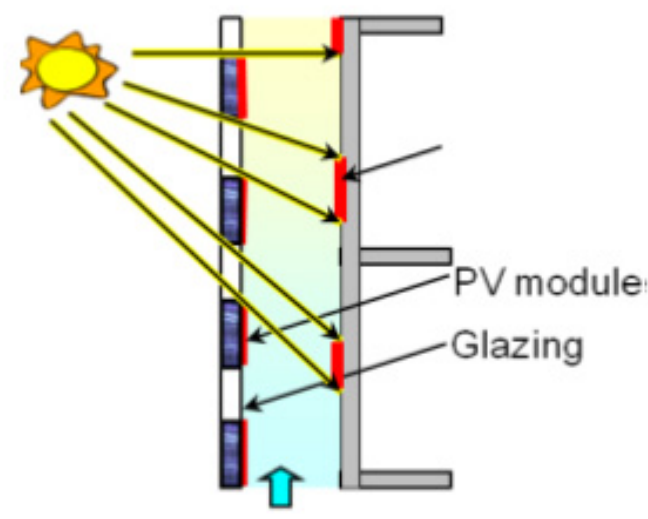

(b)

Figure 1. Schematic diagram of double-skin system (a) and staggered configuration (b).

\section{Experimental methods}

An open-ended channel built in CETHIL, Lyon, considered in this study (see Figure 2), includes two walls with height $\mathrm{H}=1.5 \mathrm{~m}$ and depth $\mathrm{L}=0.7 \mathrm{~m}$ located at the distance $\mathrm{D}=0.1 \mathrm{~m}$ apart and a $30^{\circ}$ chamfer at the channel inlet. The lateral sides are closed by plexiglass sheets. The vertical walls are insulated with thick polyurethane blocks whose thermal conductivity is $0.027 \mathrm{~W} / \mathrm{m} \mathrm{K}$. In order to mimic the heat transmitted from the solar cells, inside, the channel walls are covered by 15 independently controlled stainless steel foils, each being $10 \mathrm{~cm}$ wide and $50 \mu \mathrm{m}$ thick. Up to $500 \mathrm{~W} / \mathrm{m}^{2}$ can be supplied to the foils by the external electric generator. The emissivity of each foil is 0.092 and the thermal conductivity is $13 \mathrm{~W} / \mathrm{m} \mathrm{K}$. The channel is positioned vertically with the channel inlet above the floor and the channel outlet below an artificial ceiling.

Standard contact measurement sensors are selected to conduct the temperature measurements. The 205 thermocouples located in the plates are divided in 5 groups in such way that this type of arrangement gives 5 measurements of temperature per heating band and limits the risk of disturbing the flow. Lateral temperatures are measured through 16 thermocouples fitted in wall S1 between the central plane and the lateral edge of the channel and at 5 different vertical levels in order to monitor the horizontal variation on the heated surface temperature and evaluate the lateral conduction losses in the $\mathrm{z}$-direction.

A total of 15 thermocouples is also arranged which they are embedded inside the insulating material of each wall from top to bottom of the channel (one per heating strip, $4 \mathrm{~cm}$ deep in the insulation), to monitor the heat losses.

Instantaneous velocity fields at different locations of the channel were captured using a Particle Image Velocimetry (PIV) technique. Particles of silicon oil DEHS (Di-Ethyl-Hexyl-Sebacat) of diameter lower than $1 \mu \mathrm{m}$ and of mean density of $912 \mathrm{~kg} / \mathrm{m}^{3}$ were injected. In order to ensure a good tracking of both, the fluid motion and fluctuations, the size of the particles has been selected for a slip velocity error of $0.1 \%$ relative to the instantaneous local velocity and for a cut-off frequency around $8 \mathrm{kHz}$.

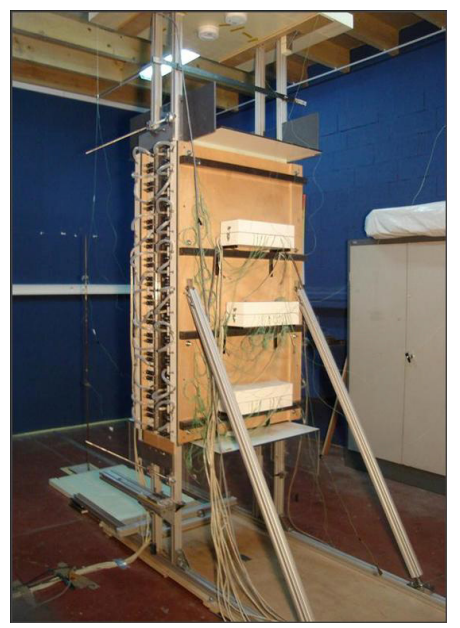

Figure 2. Open-ended channel in CETHIL, Lyon.

The acquisition of the PIV images has been done utilizing, a CCD camera with a resolution of 10-bits and a frame grabber whose acquisition frequency is of $11 \mathrm{~Hz}$. The CCD consisted of an array of 1024 x 1024 pixels, with a square pixel of size $7.4 \mu \mathrm{m}$. Each field of view represents a section of the flow of $200 \times 200 \mathrm{~mm}^{2}$, with the camera located at a distance of $0.9 \mathrm{~m}$ of the object plane. The magnitude in the uncertainty of the mean velocity is around 5\%. Experimental measurements technics as well as experimental methodology are detailed in $[4,9]$.

\section{Numerical methodologies}

The configuration investigated in this study is the double-skin façade integrated with PV modules on one side in both uniform and non-uniform heating configurations (figure 1). Buoyancy is the only driving mechanism of the flow in the channel; no mechanical ventilation is provided. 
The model previously developed in [3] has been adopted in this study. It involves a transient LES simulation using finite volume and pressure-correction methods. The model has been implemented in an inhouse Fortran code and is capable of imposing fluctuating boundary conditions at the beginning of each time step to simulate the effects of external disturbances on the flow. The model involves solution of the Favre-filtered [10] continuity, momentum, and energy conservation equations.

$$
\begin{gathered}
\frac{\partial \bar{\rho}}{\partial t}+\frac{\partial\left(\bar{\rho} \tilde{u}_{j}\right)}{\partial x_{j}}=0 \\
\frac{\partial\left(\bar{\rho} \tilde{u}_{j}\right)}{\partial t}+\frac{\partial\left(\bar{\rho} \tilde{u}_{i} \tilde{u}_{j}\right)}{\partial x_{i}}=-\frac{\partial \bar{p}}{\partial x_{j}}+\frac{\partial \tilde{\sigma}_{i j}}{\partial x_{i}}+\frac{\partial M_{i j}}{\partial x_{i}}+\left(\bar{\rho}-\rho_{r e f}\right) \vec{g} \\
\bar{\rho} C_{p} \frac{\partial \tilde{T}}{\partial t}+\bar{\rho} C_{p} \tilde{u}_{i} \frac{\partial \tilde{T}}{\partial x_{i}}=\frac{\partial}{\partial x_{i}}\left(\frac{\mu C_{p}}{\operatorname{Pr}} \frac{\partial \tilde{T}}{\partial x_{i}}\right)+\frac{\partial \bar{\tau}_{u, T}}{\partial x_{i}}
\end{gathered}
$$

where $\bar{\rho}$ and $\widetilde{T}$ are the filtered density and temperature respectively, while $\rho_{\text {ref }}$ is the reference density at the reference temperature $T_{r e f}, \widetilde{u}_{i}$ is the filtered velocity in the $i^{\text {th }}$ direction, $\bar{p}$ is the pressure, $\operatorname{Pr}$ is the molecular Prandtl number and $\mu$ is the dynamic viscosity. Here, the symbol $(\sim)$ represents a density-weighted filtering operation and the overbar $(-)$ represents a grid-filtered quantity. In Eq. (2), $\tilde{\sigma}_{i j}$ is the filtered stress tensor which, using the Stokes' hypothesis, can be written as

$$
\tilde{\sigma}_{i j}=\mu(T)\left(\frac{\partial \tilde{u}_{i}}{\partial x_{j}}+\frac{\partial \tilde{u}_{j}}{\partial x_{i}}\right)-\frac{2}{3} \mu(T) \frac{\partial \tilde{u}_{k}}{\partial x_{k}} \delta_{i j}(4)
$$

The dynamic viscosity which appears in Eq. (4) is evaluated from the Sutherland formula, viz,

$$
\mu=18.27 \times 10^{-6} \frac{T+C}{291.15+C}\left(\frac{T}{291.15}\right)^{3 / 2}
$$

where $C$ is the Sutherland constant. In the present investigation, $C$ and $P r$ are prescribed values of 120 and 0.7 respectively for air.

The terms $M_{i j}$ and $\bar{\tau}_{u, T}$ in Eq. (2) and Eq. (3) represent the unknown SGS stress tensor and the SGS heat flux vector obtained when the Favre filtering operation is applied to the equations. To obtain these terms the Vreman subgrid-scale model is adopted as it has been shown to be superior in capturing both the timeaveraged and instantaneous components of the temperature and velocity fields than other subgrid-scale models [11]. In earlier work [3], this model has also been validated against experimental data for a uniformly heated open channel [12]. Details of this model can be found in $[3,13]$. As shown in figure 2, the computational domain is extended at the channel inlet and outlet. This has been done as past research suggests that these regions influence flow features within the channel and should be included into computational models to properly model the entrainment of the flow.

For the fluid flow a stress-free boundary condition [14] is imposed at the openings (thereby allowing natural entrainment of ambient fluid. A no-slip boundary condition is enforced on all solid walls. The electrical power input to each of the foils was measured, however, the power input from each of the foils to the fluid, $\mathrm{q}_{\mathrm{in}}$, was estimated by subtracting the rate heat transfer by radiation, $\mathrm{q}_{\mathrm{rad}}$, and conduction though the insulation, $\mathrm{q}_{\text {cond }}$.

The natural convective flow in the open-ended channel is highly sensitive to external ambient conditions as natural disturbances may be introduced through the inlet section and amplified in the channel. In this work, to simulate the effect of external disturbances on the flow in the channel fluctuating velocity boundary conditions have been imposed at the channel inlet in the form shown below:

$$
u_{i}^{n}=u_{i}^{n-1}+A \cdot \mathcal{E}, \quad-1 \leq \varepsilon \leq 1
$$

in which is amplitude of disturbance and is a random number.

For the transient numerical simulation, the computational domain has been meshed using a rectangular mesh. With the channel dimensions being $0.1 \mathrm{~m} \times 1.54 \mathrm{~m} \times 0.7 \mathrm{~m}$, inside of the channel, the mesh dimensions were $41 \times 330 \times 79$; it is uniform in $x$ and $y$ directions with step sizes of $2 \mathrm{~mm}$ and $5 \mathrm{~mm}$, respectively, and non-uniform in $z$ direction with step size from $2.5 \mathrm{~mm}$ at the boundaries to $12 \mathrm{~mm}$ at the center of the channel. The inclined chamfers at the channel inlet have also been approximated using rectangular volumes. The mesh had already been shown to be adequate in a mesh convergence study Lau et al [5] for an open-ended channel configuration [12] with similar dimensions.

The governing equations for a variable-property Newtonian flow are outlined by Eq. (2) to (4) and were solved numerically by using an in-house Navier-Stokes 
LES solver. It is a robust finite volume solver which is capable in handling buoyancy-driven flows and has been validated against many numerical and experimental studies [3, 5, 12]. A transient analysis was performed with a total of 60000 time steps. The quasi-steady state was verified by observing the development of the transport variables by means of several sampling points, thereby ensuring that all variables had converged sufficiently before statistical analysis was performed on the numerical results.

\section{Results and discussion}

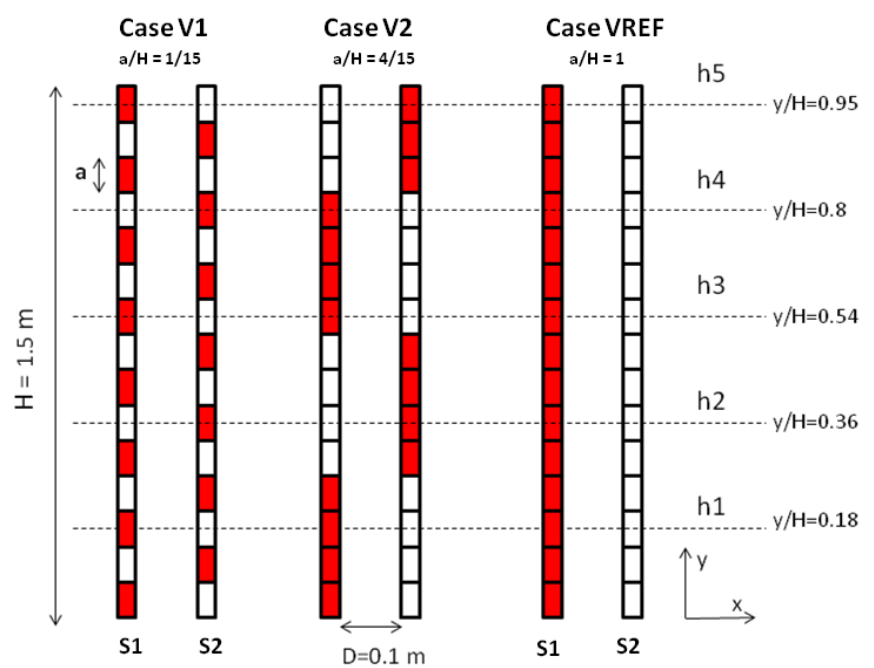

Figure 3. Studied configurations.

The effects of the non-uniformity of the wall heat flux distribution on the temperature and the velocity fields in the open ended channel, as well as on the heat transfer efficiency, were explored both numerically and experimentally for an aspect ratio of $\mathrm{D} / \mathrm{H}=1 / 15$ and heater sizes of $\mathrm{a} / \mathrm{H}=1 / 15$ and $\mathrm{a} / \mathrm{H}=4 / 15$ (cases $\mathrm{V} 1$ and $\mathrm{V} 2$ in Figure 3 with an average electrical heat input $\mathrm{P}_{\text {elec }} \approx 220$ $\mathrm{W} / \mathrm{m}^{2}$. These staggered configurations were compared against the case of one uniformly heated wall designated as VREF in figure3.

As can be seen from the temperature profiles shown in figure 4 for the non-uniformly heated configurations (Cases V1-V2), the temperature distribution displays spatial periodicity, corresponding to the alternation of the heated and unheated zones. The surface temperature rises sharply in the heated zones from the minimum value at the leading edge of the heated zone to the maximum value at the trailing edge, and then decreases along the unheated zones. It can be seen that the convective heat transfer improves for periodic heating arrangements comparing with uniform heating one, Case VREF indicated by grey lines in Figure4. The lowest average temperature is achieved for Case $\mathrm{V} 1$, with reductions up to $10^{\circ} \mathrm{C}$ at the wall S1compared to Case VREF ( Figure 4(a)). It should be noted that in Case VREF only one wall $\mathrm{S} 1$ was heated, the wall S2 was assumed to be an adiabatic.

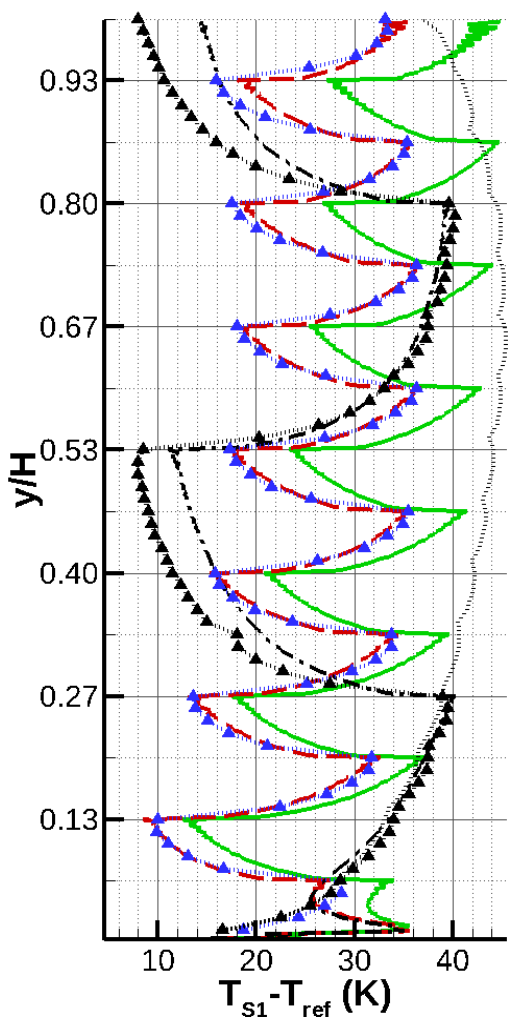

(a) S1 wall

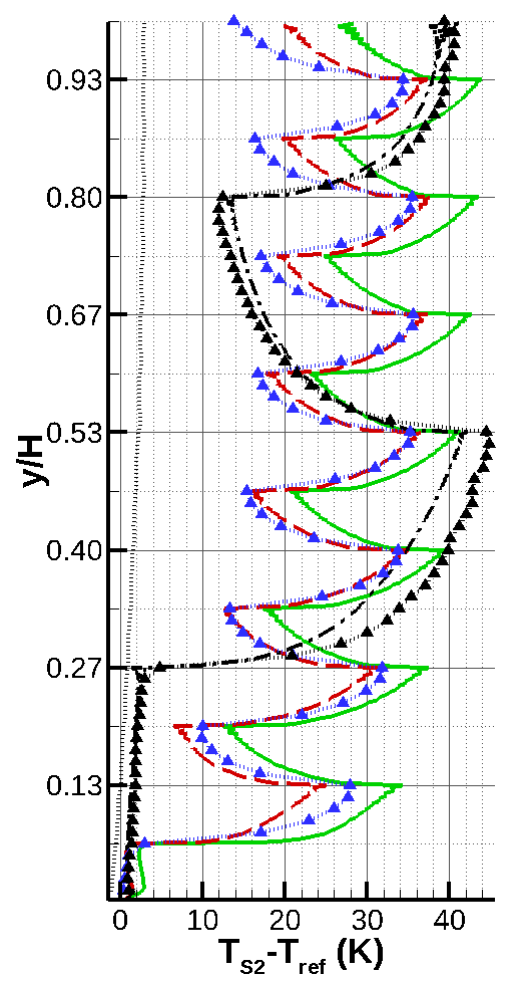

(b) S2 wall

Figure 4. Temperature along height at midline of the wall: -0 disturbances V1 numerical; - - 0.02 disturbances V1 numerical; || $\boldsymbol{\Delta} \mid$ | Experimental V1; | | Experimental VREF; || Experimental V2; --- 0.02 disturbances V2 numerical. 



(a) Case V1

(b) Case V2

Figure 5. Streamwise time-averaged velocity $V_{y}$ for cases $V 1$ and V2 at different heights of the channel: - - $\quad 0$ disturbances; - 0.02 disturbances; $\Delta$ Experimental. 
The effect of naturally occurring environmental disturbances on the temperature field is illustrated by the profiles of wall temperature for the numerical cases with different magnitude of imposed disturbances: $\mathrm{A}=0$ (no disturbances) and $A=0.02 \mathrm{~m} / \mathrm{s}$ in figure 4 . The case with disturbance produces wall temperatures which are in much better agreement with experimental data at both walls S1 and S2.

The time-averaged velocity distributions at different heights of the channel are shown in figure 5 for V1 and V2 configurations.

As can be seen from figure 5 the time-averaged velocity distributions near the inlet section $(Y / H=0.18)$ present a quasi-flat shape across the channel width. As the flow moves upwards, the velocity profiles change in shape with maximum values achieved in the vicinity of each wall. For all cases, the velocity peaks are found to increase with the height of the channel (y-direction) with values of these peaks larger in case V1 comparing to V2.

In the figure, it is also observed that there is a significant reduction of the velocity, particularly in the core region. This can be linked to a large difference of density between two layers, one near the wall that receives energy from the heated wall and one in the centre of the channel that is seems not to be affected by the injected heat. As a consequence, the near wall zones of the flow benefit from a chimney effect more than the central part of the flow. The shearing effect is thus important between these zones.

In the upper part of the channel both experimental and numerical results with imposed fluctuation indicate better mixing, with the streamwise velocity in the core region increased.

The mean mass flow rates per unit width of the channel $(\mathrm{kg} /(\mathrm{m} \mathrm{s}))$ at $\mathrm{y} / \mathrm{H}=0.54$ are presented in Figure6. Since no temperature measurements were carried out inside the channel during the experiment, to estimate mass flow rate the density of the air was evaluated based on the mean temperature between the wall temperatures of $\mathrm{S} 1$ and $\mathrm{S} 2$ which led to $21 \%$ uncertainty [9]. The numerical results, unlike the experimental results, are based on three-dimensional data, with integration of mass flow rate over the plane $\mathrm{y} / \mathrm{H}=0.54$, taking temperature and velocity at each point of the field into account which explains the difference in the results shown in the figure.

As may be observed in figure 6 the mass flow rate increases for the Cases V1-V2 comparing with Case VREF. In particular, for periodically heated V1 configuration the mass flow rate achieved is enhanced by $9 \%-12 \%$ when compared to the uniformly heated configuration. It is apparent from the analysis of the induced mass flow rate that a differentially heated channel is beneficial for ventilation while simultaneously improving the heat transfer process. The resulting increase in chimney effect is an important outcome not only in terms of cooling of PV components, but also for natural ventilation of the building. In reviewing the literature, no experimental data regarding mass flow rates were found for this type of configurations.

\section{CONCLUSIONS}

An experimental and three-dimensional computational study of natural convection in a vertical open-ended channel has been conducted for uniform and staggered, non-uniform heating configurations.

It is shown that the introduction of alternating heated and unheated zones leads simultaneously to the enhancement of convective heat transfer at the heated source level (lower wall temperature) and to an increase of the chimney effect (induced mass flow rate). In particular, for a Joule power of $233 \mathrm{~W}$, we have observed in Cases V1 and V2, a typical reduction of $10^{\circ} \mathrm{C}$ in the averaged wall temperature and an increase of mass flow rate of $9-12 \%$ with respect to the uniformly heated wall (Case VREF). These results indicate that the maximum temperature achieved at the walls is strongly related to the spatial distribution of the wall heat flux in the walls.

Thereby, in practical situations the choice of PV modules distribution on the façade appears to be of great importance as regards to the thermal behavior of the wall. On the other hand, even if the mass flow rate, which is induced in the channel for the same heat injection configuration (uniform, non-uniform) is governed by the global heat flux that is injected in the channel, it is found that it is also depending of wall heat flux spatial distribution.

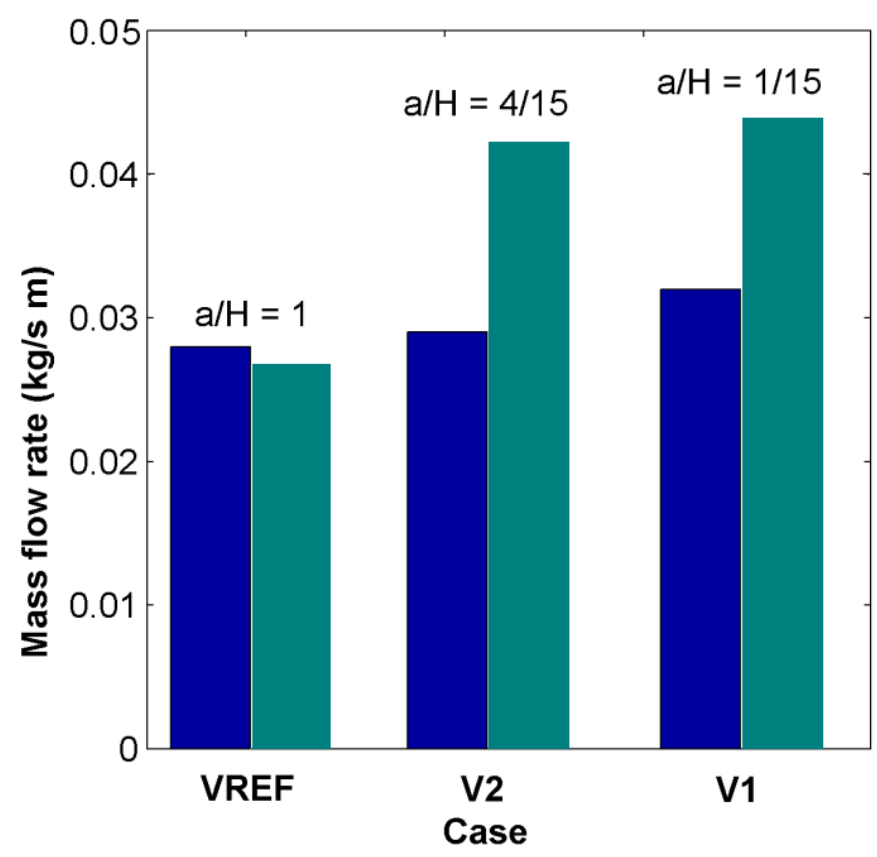

Figure 6. Mean mass flow rates per unit width of the channel $(\mathrm{kg} /(\mathrm{m} \mathrm{s}))$ at $\mathrm{y} / \mathrm{H}=0.54$, blue - experimental, green - numerical results. 


\section{References}

1. J. J. Bloem, Evaluation of a PV-integrated building application in a well-controlled outdoor test environment, Building and Environment, 43, 205-16, (2008).

2. M. Sandberg, B. Moshfegh, Buoyancy-induced air flow in photovoltaic facades: Effect of geometry of the air gap and location of solar cell modules, Building Environment. 37 (2002).

3. G.E. Lau, G.H. Yeoh, V. Timchenko, J.A. Reizes, Large-eddy simulation of turbulent natural convection in vertical parallel-plate channels, Numerical Heat Transfer, Part B Fundamentals. 59(4) 259-287, (2011).

4. E. Sanvicente, S. Giroux-Julien, C. Ménézo, H. Bouia, Transitional natural convection flow and heat transfer in an open channel, International Journal Thermal Sciences. 63, 87-104, (2013)

5. G.E. Lau, G.H. Yeoh, V. Timchenko, J.A. Reizes, Numerical investigation of passive cooling in open vertical channels, Applied Thermal Engineering, 39, 121-131, (2012).

6. M. Fossa, C. Ménézo, E. Leonardi, Experimental natural convection on vertical surfaces for building integrated photovoltaic (BIPV) applications, Experimental Thermal Fluid Science. 32(4), 980-990, (2008).

7. J. Vareilles, "Etude des transferts de chaleur dans un canal vertical différentiellement chauffé: application aux enveloppes photovoltaïques-thermique," Lyon 1 University, Lyon, (2007).
8. S. Giroux-Julien, C. Ménézo, J. Vareilles, H. Pabiou, M. Fossa, E. Leonardi, Natural convection in nonuniformly heated channel with application to photovoltaic facades, Computational thermal Sciences. 1(3), 231-258, (2009).

9. E. Sanvicente, Experimental investigation of thermal and fluid dynamical behavior of flows in open-ended channels: Application to Building Integrated Photovoltaic (BiPV) Systems, Centre Thermique de Lyon (CETHIL) / CFD Laboratory (UNSW), (2013)

10. A. Favre, "Equations statistiques aux fluctuations turbulentes dans les ecoulements compressibles: cas des vitesses et des temperatures," C R Acad Sci Paris, 273, (1971)

11. G. Lau, G. Yeoh, V. Timchenko and J. Reizes, "Large-eddy simulation of natural convection in an asymmetrically-heated vertical parallel-plate channel: Assessment of subgrid-scale models," Computers \& Fluids, 59, 101-116, (2012).

12. M. Miyamoto, Y. Katoh and H. Sasaki, "Turbulent free convection heat transfer from vertical parallel plates," in Eighth International Heat Transfer Conference, Washington, DC, (1986).

13. G. Lau, E. Sanvicenteb, G. Yeoh, V. Timchenko, M. Fossa, C. Menezo and S. Giroux-Julien, "Modelling of natural convection in vertical and tilted photovoltaic applications," Energy And Buildings, 55, 810-822, (2012).

14. T. Papanastasiou, N. Malamataris and K. Ellwood, "A new outflow boundary condition," International Journal for Numerical Methods in Fluids, 14, $587-$ 608, (1992). 